\title{
Topological Superconductivity and Superfluidity
}

\author{
Xiao-Liang Qi, Taylor L. Hughes, Srinivas Raghu and Shou-Cheng Zhang \\ Department of Physics, McCullough Building, Stanford University, Stanford, CA 94305-4045
}

\begin{abstract}
We construct time reversal invariant topological superconductors and superfluids in two and three dimensions which are analogous to the recently discovered quantum spin Hall and three-d $Z_{2}$ topological insulators respectively. These states have a full pairing gap in the bulk, gapless counterpropagating Majorana states at the boundary, and a pair of Majorana zero modes associated with each vortex. We show that the time reversal symmetry naturally emerges as a supersymmetry, which changes the parity of the fermion number associated with each time-reversal invariant vortex. In the presence of external T-breaking fields, non-local topological correlation is established among these fields, which is an experimentally observable manifestation of the emergent supersymmetry.

PACS numbers: 74.20.Rp, 73.43.-f, 67.30.he, 74.45.+c
\end{abstract}

The search for topological states of quantum matter has become an active and exciting pursuit in condensed matter physics. The quantum Hall (QH) effect [1] provides the first example of a topologically non-trivial state of matter, where the quantized Hall conductance is a topological invariant 2]. Recently, the quantum spin Hall (QSH) state 3, 4] has been theoretically predicted [5] and experimentally observed in HgTe quantum well systems [6]. The time reversal invariant (TRI) QSH state is characterized by a bulk gap, a $Z_{2}$ topological number [7], and gapless helical edge states, where time-reversed partners counter-propagate $[\underline{6},[$ ] $]$.

Chiral superconductors in a time reversal symmetry breaking (TRB) $\left(p_{x}+i p_{y}\right)$ pairing state in $2 \mathrm{~d}$ have a sharp topological distinction between the strong and weak pairing regimes [10]. In the weak pairing regime, the system has a full bulk gap and gapless chiral Majorana states at the edge, which are topologically protected. Moreover, a Majorana zero mode is trapped in each vortex core [10], which leads to a ground state degeneracy of $2^{n-1}$ in the presence of $2 n$ vortices. When the vortices wind around each other a non-Abelian Berry phase is generated in the $2^{n-1}$ dimensional ground state manifold, which implies non-Abelian statistics for the vortices [11]. Chiral superconductors are analogous to the QH state - they both break time reversal (TR) and have chiral edge states with linear dispersion. However, the edge states of a chiral superconductor have only half the degrees of freedom compared to the $\mathrm{QH}$ state, since the negative energy quasiparticle operators on the edge of a chiral superconductor describe the same excitations as the positive energy ones.

Given the analogy between the chiral superconducting state and the QH state, and with the recent discovery of the TRI QSH state, it is natural to generalize the chiral pairing state to the helical pairing state, where fermions with up spins are paired in the $\left(p_{x}+i p_{y}\right)$ state, while fermions with down spins are paired in the $\left(p_{x}-i p_{y}\right)$ state. Such a TRI state have a full gap in the bulk, and counter-propagating helical Majorana states at the edge (in contrast, the edge states of the TRI topological insulator are helical Dirac fermions). Just as in the case of the QSH state, a mass term for an odd number of pairs of helical Majorana states is forbidden by TR symmetry, and therefore, a topologically protected superconducting or superfluid state can exist in the presence of time-reversal symmetry. Recently, a $Z_{2}$ classification of the topological superconductor has been discussed in Refs [12, 13, 14], by noting the similarity between the Bogoliubov-de Gennes (BdG) superconductor Hamiltonian and the QSH insulator Hamiltonian. The four types of topological states of matter discussed here are summarized in Fig. 11. In this work, we give a $Z_{2}$ classification of both the $2 \mathrm{D}$ and $3 \mathrm{D}$ cases which has a profound physical implication. In two dimensions, we show that a time-reversal invariant topological defect of a $Z_{2}$ nontrivial superconductor carries a Kramers' pair of Majorana fermions. Let $N_{F}$ be the operator which measures the number of fermions of a general system, then the fermion-number parity operator is given by $(-1)^{N_{F}}$. This operator is also referred to as the Witten index 15], which plays a crucial role in supersymmetric theories. We prove the remarkable fact that in the presence of a topological defect, the TR operator $\mathcal{T}$ changes the fermion number parity, $\mathcal{T}^{-1}(-1)^{N_{F}} \mathcal{T}=-(-1)^{N_{F}}$ locally around the defect in the $Z_{2}$ non-trivial state, while it preserves the fermion number parity, $\mathcal{T}^{-1}(-1)^{N_{F}} \mathcal{T}=(-1)^{N_{F}}$, in the $Z_{2}$ trivial state. This fact gives a precise definition of the $Z_{2}$ topological classification of any TRI superconductor state and is generally valid in the presence of interactions and disorder. A supersymmetric operation can be defined as an operation which changes the fermion number parity; therefore, in this precise sense, we show that the TR symmetry emerges as a supersymmetry in topological superconductors. Though supersymmetry has been studied extensively in high energy physics, it has not yet been observed in Nature. Our proposal offers the opportunity to experimentally observe supersymmetry in condensed matter systems without any fine tuning of microscopic parameters. The physical consequences of such a supersymmetry is also studied.

As the starting point, we consider a TRI $p$-wave superconductor with spin triplet pairing, which has the fol-

SIMES, SLAC National Accelerator Center, 2575 Sand Hill Road, Menlo Park, CA 94309

Work supported in part by US Department of Energy contract DE-AC02-76SF00515. 


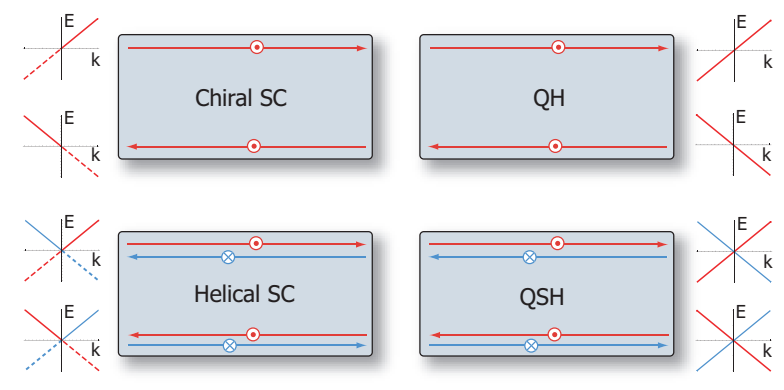

FIG. 1: (Top row) Schematic comparison of $2 d$ chiral superconductor and the QH state. In both systems, TR symmetry is broken and the edge states carry a definite chirality. (Bottom row) Schematic comparison of $2 d$ TRI topological superconductor and the QSH insulator. Both systems preserve TR symmetry and have a helical pair of edge states, where opposite spin states counter-propagate. The dashed lines show that the edge states of the superconductors are Majorana fermions so that the $E<0$ part of the quasi-particle spectra are redundant. In terms of the edge state degrees of freedom, we have $(\mathrm{QSH})=(\mathrm{QH})^{2}=(\text { Helical SC })^{2}=(\text { Chiral SC })^{4}$.

lowing $4 \times 4$ BdG Hamiltonian:

$$
H=\frac{1}{2} \int d^{2} x \Psi^{\dagger}(x)\left(\begin{array}{cc}
\epsilon_{\mathbf{p}} \mathbb{I} & i \sigma_{2} \sigma_{\alpha} \Delta^{\alpha j} p_{j} \\
\text { h.c. } & -\epsilon_{\mathbf{p}} \mathbb{I}
\end{array}\right) \Psi(x)
$$

with $\Psi(x)=\left(c_{\uparrow}(x), c_{\downarrow}(x), c_{\uparrow}^{\dagger}(x), c_{\downarrow}^{\dagger}(x)\right)^{T}, \epsilon_{\mathbf{p}}=\mathbf{p}^{2} / 2 m-$ $\mu$ the kinetic energy and chemical potential terms and h.c. $\equiv\left(i \sigma_{2} \sigma_{\alpha} \Delta^{\alpha j} p_{j}\right)^{\dagger}$. The TR transformation is defined as $c_{\uparrow} \rightarrow c_{\downarrow}, \quad c_{\downarrow} \rightarrow-c_{\uparrow}$. It can be shown that the Hamiltonian (11) is time-reversal invariant if $\Delta_{\alpha j}$ is a real matrix. To show the existence of a topological state, consider the TRI mean-field ansatz $\Delta^{\alpha 1}=\Delta(1,0,0), \Delta^{\alpha 2}=$ $\Delta(0,1,0)$. For such an ansatz the Hamiltonian (1) is block diagonal with only equal spin pairing:

$$
H=\frac{1}{2} \int d^{2} x \tilde{\Psi}^{\dagger}\left(\begin{array}{cccc}
\epsilon_{\mathbf{p}} & \Delta p_{+} & & \\
\Delta p_{-} & -\epsilon_{\mathbf{p}} & & \\
& & \epsilon_{\mathbf{p}} & -\Delta p_{-} \\
& & -\Delta p_{+} & -\epsilon_{\mathbf{p}}
\end{array}\right) \tilde{\Psi}
$$

with $\tilde{\Psi}(x) \equiv\left(c_{\uparrow}(x), c_{\uparrow}^{\dagger}(x), c_{\downarrow}(x), c_{\downarrow}^{\dagger}(x)\right)^{T}$, and $p_{ \pm}=$ $p_{x} \pm i p_{y}$. From this Hamiltonian we see that the spin up (down) electrons form $p_{x}+i p_{y}\left(p_{x}-i p_{y}\right)$ Cooper pairs, respectively. In the weak pairing phase with $\mu>0$, the $\left(p_{x}+i p_{y}\right)$ chiral superconductor is known to have chiral Majorana edge states propagating on each boundary, described by the Hamiltonian $H_{\text {edge }}=$ $\sum_{k_{y} \geq 0} v_{F} k_{y} \psi_{-k_{y}} \psi_{k_{y}}$, where $\psi_{-k_{y}}=\psi_{k_{y}}^{\dagger}$ is the quasiparticle creation operator [10] and the boundary is taken to be parallel to the $y$ direction. Thus we know that the edge states of the TRI system described by Hamiltonian (2) consist of spin up and spin down quasi-particles with opposite chirality:

$$
H_{\text {edge }}=\sum_{k_{y} \geq 0} v_{F} k_{y}\left(\psi_{-k_{y} \uparrow} \psi_{k_{y} \uparrow}-\psi_{-k_{y} \downarrow} \psi_{k_{y} \downarrow}\right) .
$$

The quasi-particle operators $\psi_{k_{y} \uparrow}, \psi_{k_{y} \downarrow}$ can be expressed in terms of the eigenstates of the BdG Hamiltonian as

$$
\begin{aligned}
\psi_{k_{y} \uparrow} & =\int d^{2} x\left(u_{k_{y}}(x) c_{\uparrow}(x)+v_{k_{y}}(x) c_{\uparrow}^{\dagger}(x)\right) \\
\psi_{k_{y} \downarrow} & =\int d^{2} x\left(u_{-k_{y}}^{*}(x) c_{\downarrow}(x)+v_{-k_{y}}^{*}(x) c_{\downarrow}^{\dagger}(x)\right)
\end{aligned}
$$

from which the time-reversal transformation of the quasiparticle operators can be determined to be $\mathcal{T}^{-1} \psi_{k_{y} \uparrow} \mathcal{T}=$ $\psi_{-k_{y} \downarrow}, \quad \mathcal{T}^{-1} \psi_{k_{y} \downarrow} \mathcal{T}=-\psi_{-k_{y} \uparrow}$. In other words, $\left(\psi_{k_{y} \uparrow}, \psi_{-k_{y} \downarrow}\right)$ transforms as a Kramers' doublet, which forbids a gap in the edge states due to mixing of the spin-up and spin-down modes when TR is preserved. To see this explicitly, notice that the only $k_{y}$-independent term that can be added to the edge Hamiltonian (3) is $i m \sum_{k_{y}} \psi_{-k_{y} \uparrow} \psi_{k_{y} \downarrow}$ with $m \in \mathbb{R}$. However, such a term is odd under TR, which implies that any back scattering between the quasi-particles is forbidden by TR symmetry. The discussion above is exactly parallel to the $Z_{2}$ topological characterization of the quantum spin Hall system. In fact, the Hamiltonian (2) has exactly the same form as the four band effective Hamiltonian proposed in Ref. [5] to describe HgTe quantum wells with the QSH effect. The edge states of the QSH insulators consist of an odd number of Kramers' pairs, which remain gapless under any small TR-invariant perturbation [8, [9]. A no-go theorem states that such a "helical liquid" with an odd number of Kramers' pairs at the Fermi energy can not be realized in any bulk 1d system, but can only appear as an edge theory of a $2 \mathrm{~d}$ QSH insulator 9 ]. Similarly, the edge state theory (3) can be called a "helical Majorana liquid", which can only exist on the boundary of a $Z_{2}$ topological superconductor. Once such a topological phase is established, it is robust under any TRI perturbations.

The Hamiltonian (10) can be easily generalized to three dimensions, in which case $\Delta^{\alpha j}$ becomes a $3 \times 3$ matrix with $\alpha=1,2,3$ and $j=x, y, z$. An example of such a Hamiltonian is given by the well-known ${ }^{3} \mathrm{He}$ BW phase, for which the order parameter $\Delta^{\alpha j}$ is determined by an orthogonal matrix $\Delta^{\alpha j}=\Delta u^{\alpha j}, u \in \mathrm{SO}(3)[16]$. Here and below we ignore the dipole-dipole interaction term [17] since it does not affect any essential topological properties. By applying a spin rotation, $\Delta^{\alpha j}$ can be diagonalized to $\Delta^{\alpha j}=\Delta \delta^{\alpha j}$, in which case the Hamiltonian (11) has the same form as a 3d Dirac Hamiltonian with momentum dependent mass $\epsilon(\mathbf{p})=\mathbf{p}^{2} / 2 m-\mu$. We know that a band insulator described by the Dirac Hamiltonian is a $3 \mathrm{~d} Z_{2}$ topological insulator for $\mu>0$ [18, 19, 20], and has nontrivial surface states. The corresponding superconductor Hamiltonian describes a topological superconductor with $2 \mathrm{~d}$ gapless Majorana surface states. The 
surface theory can be written as

$$
H_{\text {surf }}=\frac{1}{2} \sum_{\mathbf{k}} v_{F} \psi_{-\mathbf{k}}^{T}\left(\sigma_{z} k_{x}+\sigma_{x} k_{y}\right) \psi_{\mathbf{k}}
$$

which remains gapless under any small TRI perturbation since the only available mass term $m \sum_{\mathbf{k}} \psi_{-\mathbf{k}}^{T} \sigma_{y} \psi_{\mathbf{k}}$ is time-reversal odd. We would like to mention that the surface Andreev bound states in ${ }^{3} \mathrm{He}-\mathrm{B}$ phase have been observed experimentally [21].

To understand the physical consequences of the nontrivial topology we study the TRI topological defects of the topological superconductors. We start by considering the equal-spin pairing system with BdG Hamiltonian (2) in which spin up and down electrons form $p_{x}+i p_{y}$ and $p_{x}-i p_{y}$ Cooper pairs, respectively. A TRI topological defect can be defined as a vortex of spin-up superfluid coexisting with an anti-vortex of spindown superfluid at the same position. In the generic Hamiltonian (11), such a vortex configuration is written as $\Delta^{\alpha j}=\left[\exp \left(i \sigma_{2} \theta\left(\mathbf{r}-\mathbf{r}_{\mathbf{0}}\right)\right)\right]^{\alpha j}, \alpha=1,2$ and $\Delta^{3 j}=0$, where $\theta\left(\mathbf{r}-\mathbf{r}_{\mathbf{0}}\right)$ is the angle of $\mathbf{r}$ with respect to the vortex position $\mathbf{r}_{0}$. Since in the vortex core of a weak pairing $p_{x}+i p_{u}$ superconductor there is a single Majorana zero mode [10, 22], one immediately knows that a pair a Majorana zero modes exist in the vortex core we study here. In terms of the electron operators, the two Majorana fermion operators can be written as

$$
\begin{aligned}
& \gamma_{\uparrow}=\int d^{2} x\left(u_{0}(x) c_{\uparrow}(x)+u_{0}^{*}(x) c_{\uparrow}^{\dagger}(x)\right) \\
& \gamma_{\downarrow}=\int d^{2} x\left(u_{0}^{*}(x) c_{\uparrow}(x)+u_{0}(x) c_{\uparrow}^{\dagger}(x)\right)
\end{aligned}
$$

where we have used the fact that the spin-down zero mode wave function can be obtained from the timereversal transformation of the spin-up one. The Majorana operators satisfy the anti-commutation relation $\left\{\gamma_{\alpha}, \gamma_{\beta}\right\}=2 \delta_{\alpha \beta}$. The TR transformation of the Majorana fermions is

$$
\mathcal{T}^{-1} \gamma_{\uparrow} \mathcal{T}=\gamma_{\downarrow}, \mathcal{T}^{-1} \gamma_{\downarrow} \mathcal{T}=-\gamma_{\uparrow} .
$$

Similar to the case of the edge states studied earlier, the Majorana zero modes are robust under any small TRI perturbation, since the only possible term $i m \gamma_{\uparrow} \gamma_{\downarrow}$ which can lift the zero modes to finite energy is TR odd, i.e., $\mathcal{T}^{-1} i \gamma_{\uparrow} \gamma_{\downarrow} \mathcal{T}=-i \gamma_{\uparrow} \gamma_{\downarrow}$.

The properties of such a topological defect appear identical to that of a $\pi$-flux tube threading into a TRI topological insulator 23, 24], where a Kramers' pair of complex fermions are trapped by the flux tube. However, there is an essential difference. From the two Majorana zero modes $\gamma_{\uparrow}, \gamma_{\downarrow}$ a complex fermion operator can be defined as $a=\left(\gamma_{\uparrow}+i \gamma_{\downarrow}\right) / 2$, which satisfies the fermion anticommutation relation $\left\{a, a^{\dagger}\right\}=1$. Since $\gamma_{\uparrow}, \gamma_{\downarrow}$ are zero modes, we obtain $[a, H]=0$ which implies that $a$ is the annihilation operator of a zero-energy quasiparticle. Consequently, the ground state of the system is at least two-fold degenerate, with two states $\left|G_{0}\right\rangle$ and $\left|G_{1}\right\rangle=a^{\dagger}\left|G_{0}\right\rangle$ containing 0 and $1 a$-fermions. Since $a^{\dagger} a=\left(1+i \gamma_{\uparrow} \gamma_{\downarrow}\right) / 2$, the states $\left|G_{0(1)}\right\rangle$ are eigenstates of $i \gamma_{\uparrow} \gamma_{\downarrow}$ with eigenvalues $-1(+1)$, respectively. Thus from the oddness of $i \gamma_{\uparrow} \gamma_{\downarrow}$ under TR we know that $\left|G_{0}\right\rangle$ and $\left|G_{1}\right\rangle$ are time-reversed partners. Note that superconductivity breaks the charge $U(1)$ symmetry to $Z_{2}$, meaning that the fermion number parity operator $(-1)^{N_{F}}$ is conserved. Thus, all the eigenstates of the Hamiltonian can be classified by the value of $(-1)^{N_{F}}$. If, say, $\left|G_{0}\right\rangle$ is a state with $(-1)^{N_{F}}=1$, then $\left|G_{1}\right\rangle=a^{\dagger}\left|G_{0}\right\rangle$ must satisfy $(-1)^{N_{F}}=-1$. Since $\left|G_{0}\right\rangle$ and $\left|G_{1}\right\rangle$ are time-reversal partners, we know that in the Hilbert space of the zeroenergy states the TR transformation changes the fermion number parity:

$$
\mathcal{T}^{-1}(-1)^{N_{F}} \mathcal{T}=-(-1)^{N_{F}} .
$$

Eq. (8) is the central result of this paper. At a first glance it seems contradict the fundamental fact that the electron number of the whole system is invariant under TR. Such a paradox is resolved by noticing that there are always an even number of topological defects in a closed system without boundary. Under the TR transformation, the fermion number parity around each vortex core is odd, but the total fermion number parity remains even as expected. Once the anomalous transformation property (8) is established for a topological defect in a TRI superconductor, it is robust under any TRI perturbation as long as the bulk quasiparticle gap remains finite and other topological defects are far away. Thus Eq. (8) is a generic definition of TRI topological superconductors:

- Definition I. A two-dimensional TRI superconductor is $Z_{2}$ nontrivial if and only if fermion number parity around a TRI topological defect is odd under TR.

A transformation changing fermion number by an odd number is a "supersymmetry"; thus, the TR symmetry emerges as a discrete supersymmetry for each TRI topological defect. The same analysis applies to the edge theory (3), which shows that in the 1d helical Majorana liquid is a theory with TR symmetry as a discrete supersymmetry.

All the conclusions above can be generalized to $3 \mathrm{~d}$ topological superconductors. In the ${ }^{3} \mathrm{He} \mathrm{BW}$ phase the Goldstone manifold of the order parameter is $\Delta^{\alpha j}=$ $\Delta u^{\alpha j} \in S O(3) \times U(1)[16,25]$. A time-reversal invariant configuration satisfies $\Delta^{\alpha j} \in \mathbb{R}$, which restricts the order parameter to $S O(3)$. Since $\Pi_{1}(S O(3))=Z_{2}$, the TRI topological defects are 1d "vortex" rings. By solving the BdG equations in the presence of such vortex rings, it can be shown that there are linearly dispersing quasiparticles propagating on each vortex ring, similar 
to the edge states of the $2 \mathrm{~d}$ topological superconductor. However, for a ring with finite length the quasi-particle spectrum is discrete. Specifically, there may or may not be a pair of Majorana modes at exactly zero energy. The existence of the Majorana zero modes on the vortex rings turns out to be a topological property determined by the linking number between different vortex rings. Due to the length constraints of the present paper, we will write our conclusion and leave the details for a separate work: There are a pair of Majorana fermion zero modes confined on a vortex ring if and only if the ring is linked to an odd number of other vortex rings. Such a condition is shown in Fig. 2. Consequently, the generalization of Definition I to $3 \mathrm{~d}$ is:

- Definition II. A 3d TRI superconductor is $Z_{2}$ nontrivial if and only if the fermion number parity around one of the two mutually-linked TRI vortex rings is odd under TR. (a)

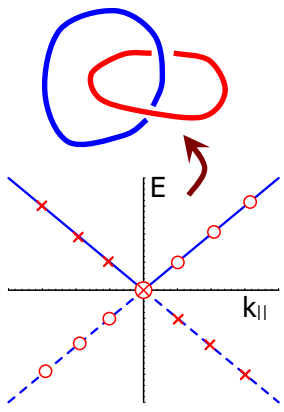

(b)

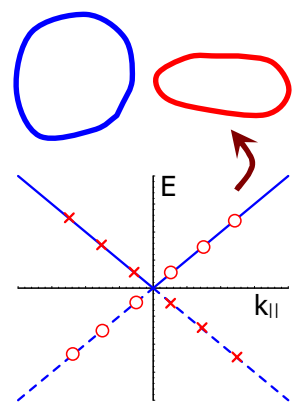

FIG. 2: Illustration of a 3d TRI topological superconductor with two TRI vortex rings which are (a) linked or (b) unlinked. The $E-k_{\|}$dispersion relations show schematically the quasiparticle levels confined on the red vortex ring in both cases. "o" and " $\times$ " stand for the quasiparticle levels that are Kramers' partners of each other. Only case (a) has a pair of Majorana zero modes located on each vortex ring.

Besides providing a generic definition of the $Z_{2}$ topological superconductors, such an emergent supersymmetry also leads to physical predictions. Consider the $2 \mathrm{~d}$ topological superconductor coupled to a weak TRbreaking field $M(\mathbf{r})$, which is classical but can have thermal fluctuations. This situation can be realized in an isolated superconductor with vortices pinned to quenched weak magnetic impurities. The $n$-point correlation function of $M(\mathbf{r})$ can be obtained by

$$
\left\langle\prod_{s=1}^{n} M\left(\mathbf{r}_{s}\right)\right\rangle \equiv \int \frac{D[M(\mathbf{r})]}{Z} \prod_{s=1}^{n} M\left(\mathbf{r}_{s}\right) \operatorname{Tr}\left(e^{-\beta H[M]}\right)_{\text {even }}
$$

in which the trace is restricted to states with an even number of fermions. For a closed system with $N$ vortices, the leading order effect of the TR-breaking field is to lift the degeneracy between the two Majorana fermions (a)

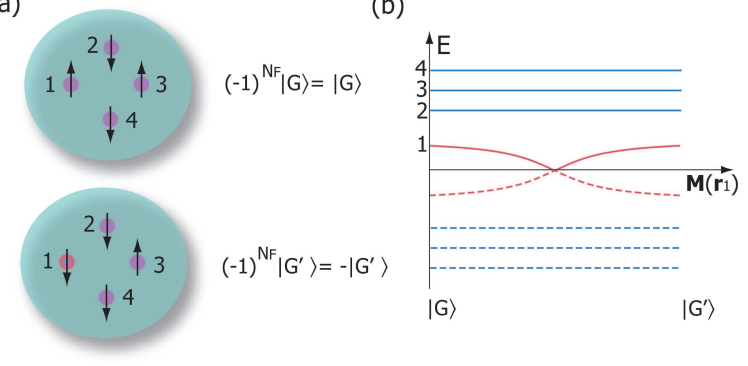

FIG. 3: (a) Illustration of a 2d TRI topological superconductor with four TRI topological defects coupled to a TRbreaking field. The arrows show the sign of the TR-breaking field $\operatorname{sgn}\left(M\left(\mathbf{r}_{s}\right)\right)$ at each topological defect. In the two configurations shown, only the field around vortex 1 is flipped, leading to an opposite fermion number parity in the corresponding ground state $|G\rangle$ and $\left|G^{\prime}\right\rangle$ (see text). (b) Illustration showing the flow of the energy levels when the upper configuration in figure (a) is deformed to the lower one. The flip of the TR-breaking field $M\left(\mathbf{r}_{1}\right)$ leads to a level crossing at $M\left(\mathbf{r}_{1}\right)=0$, where the fermion number parity in the ground state changes sign.

in each vortex core. Consequently, the perturbed Hamiltonian $H[M(\mathbf{r})]$ to first order can be written as

$$
H[M(\mathbf{r})]=\sum_{s=1}^{N} i M\left(\mathbf{r}_{s}\right) a_{s} \gamma_{s \uparrow} \gamma_{s \downarrow}
$$

in which $\gamma_{s \uparrow(\downarrow)}$ are the Majorana fermion operators, and $a_{s} \in \mathbb{R}$ depend on the details of the perturbation. The important fact is that the mass term induced is linear in $M(\mathbf{r})$ at the defect position $\mathbf{r}_{s}$, since $i \gamma_{s \uparrow} \gamma_{s \downarrow}$ is TR odd.

Since the superconductor has a full gap, naively one would expect all the correlations of $M(\mathbf{r})$ field to be short ranged. However, for a system with $N$ topological defects the $N$-point correlation function has a long range order when $\mathbf{r}_{s}, s=1,2, . . N$ are chosen to be the coordinates of the topological defects. In other words, the correlation function

$$
\lim _{\left|\mathbf{r}_{i}-\mathbf{r}_{j}\right| \rightarrow \infty, \forall i, j}\left\langle\prod_{s=1}^{N} M\left(\mathbf{r}_{s}\right)\right\rangle \neq 0,
$$

though all the $n$ point correlations in Eq. (9) with $n<N$ remain short ranged. Physically, such a non-local correlation can be understood by comparing two states $|G\rangle$ and $\left|G^{\prime}\right\rangle$, which are the ground states of the systems with the field configurations $\mathcal{M} \equiv\left(M\left(\mathbf{r}_{1}\right), M\left(\mathbf{r}_{2}\right), \ldots, M\left(\mathbf{r}_{N}\right)\right)$ and $\mathcal{M}^{\prime} \equiv\left(-M\left(\mathbf{r}_{1}\right), M\left(\mathbf{r}_{2}\right), \ldots, M\left(\mathbf{r}_{N}\right)\right)$, respectively. From Hamiltonian (10) it can be seen that $|G\rangle$ and $\left|G^{\prime}\right\rangle$ have opposite fermion number parity, since the fermion number parity around the first topological defect $i \gamma_{1 \uparrow} \gamma_{1 \downarrow}$ is reversed while that of all the other topological defects remains invariant, as shown in Fig. 3. Without loss of generality, we can assume $(-1)^{N_{F}}$ is even for $|G\rangle$ and odd 
for $\left|G^{\prime}\right\rangle$. Since the whole system is required to have an even number of fermions, the lowest energy state in the Hilbert space for the field configuration $\mathcal{M}^{\prime}$ is not $\left|G^{\prime}\right\rangle$, but the lowest quasiparticle excitation $a_{\min }^{\dagger}\left|G^{\prime}\right\rangle$. Thus, the two field configurations $\mathcal{M}$ and $\mathcal{M}^{\prime}$ have different free energies, which leads to the non-vanishing correlation function in Eq. (11). Even when the topological defects are arbitrarily far away, the energy difference between the two configurations remains finite, which shows the non-local topological correlation. Similar non-local correlations can also be obtained for a $3 \mathrm{~d}$ TRI topological superconductor with linked vortex rings.

Acknowledgement.-We acknowledge helpful discussions with S. B. Chung, A. L. Fetter, L. Fu, R. Roy and S. Ryu. This work is supported by the NSF under grant numbers DMR-0342832, the US Department of Energy, Office of Basic Energy Sciences under contract DE-AC03-76SF00515, and the Stanford Institute for Theretical Physics (S.R.).

[1] R. E. Prange and S. M. Girvin, eds., The Quantum Hall effect (Springer-Verlag, USA, 1990).

[2] D. J. Thouless, M. Kohmoto, M. P. Nightingale, and M. den Nijs, Phys. Rev. Lett. 49, 405 (1982).

[3] C. L. Kane and E. J. Mele, Phys. Rev. Lett. 95, 226801 (2005).

[4] B.A. Bernevig and S.C. Zhang, Phys. Rev. Lett. 96, 106802 (2006).

[5] B. A. Bernevig, T. L. Hughes, and S.C. Zhang, Science
314, 1757 (2006).

[6] M. König, S. Wiedmann, C. Brüne, A. Roth, H. Buhmann, L. Molenkamp, X.-L. Qi, and S.-C. Zhang, Science 318, 766 (2007).

[7] C. L. Kane and E. J. Mele, Phys. Rev. Lett. 95, 146802 (2005).

[8] C. Xu and J. Moore, Phys. Rev. B 73, 045322 (2006).

[9] C. Wu, B.A. Bernevig, and S.C. Zhang, Phys. Rev. Lett. 96, 106401 (2006).

[10] N. Read and D. Green, Phys. Rev. B 61, 10267 (2000).

[11] D. A. Ivanov, Phys. Rev. Lett. 86, 268 (2001).

[12] R. Roy, arxiv: cond-mat/0608064.

[13] R. Roy, arxiv: 0803.2868 (2008).

[14] A. P. Schnyder, S. Ryu, A. Furusaki, and A. W. W. Ludwig, e-print arXiv: 0803.2786 (2008).

[15] E. Witten, Nucl. Phys. B 202, 253 (1982).

[16] D. Vollhardt and P. Wölfle, The Superfluid Phases of Helium 3 (Taylor and Francis, USA, 1990).

[17] A. J. Leggett, Rev. Mod. Phys. 47, 331 (1975).

[18] L. Fu, C. L. Kane, and E. J. Mele, Phys. Rev. Lett. 98, 106803 (2007).

[19] J. E. Moore and L. Balents, Phys. Rev. B 75, 121306 (2007).

[20] R. Roy, arxiv: cond-mat/0607531.

[21] Y. Aoki, Y. Wada, M. Saitoh, R. Nomura, Y. Okuda, Y. Nagato, M. Yamamoto, S. Higashitani, and K. Nagai, Phys. Rev. Lett. 95, 075301 (2005).

[22] M. Stone and S.-B. Chung, Phys. Rev. B 73, 014505 (2006).

[23] X. L. Qi and S. C. Zhang, arxiv: cond-mat/0801.0252.

[24] Y. Ran, A. Vishwanath, and D.-H. Lee, arxiv: condmat/0801.0627.

[25] M. M. Salomaa and G. E. Volovik, Rev. Mod. Phys. 59, 533 (1987). 